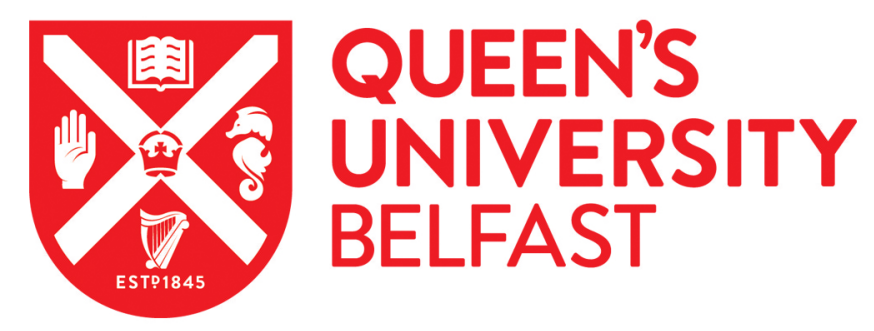

\title{
A qualitative study of community pharmacists' awareness of and involvement with intermediate care facilities
}

Millar, A. N., Hughes, C. M., \& Ryan, C. (2016). A qualitative study of community pharmacists' awareness of and involvement with intermediate care facilities. European Journal for Person Centered Healthcare, 4(1), 53-60. https://doi.org/10.5750/ejpch.v4i1.1063

\section{Published in:}

European Journal for Person Centered Healthcare

Document Version:

Publisher's PDF, also known as Version of record

Queen's University Belfast - Research Portal:

Link to publication record in Queen's University Belfast Research Portal

Publisher rights

(C) 2016 The Authors.

\section{General rights}

Copyright for the publications made accessible via the Queen's University Belfast Research Portal is retained by the author(s) and / or other copyright owners and it is a condition of accessing these publications that users recognise and abide by the legal requirements associated with these rights.

Take down policy

The Research Portal is Queen's institutional repository that provides access to Queen's research output. Every effort has been made to ensure that content in the Research Portal does not infringe any person's rights, or applicable UK laws. If you discover content in the Research Portal that you believe breaches copyright or violates any law, please contact openaccess@qub.ac.uk. 


\title{
A qualitative study of community pharmacists' awareness of and involvement with intermediate care facilities
}

\author{
Anna N. Millar MPharm MPSNI ${ }^{a}$, Carmel M. Hughes BSc PhD MRPharmS MPSNI ${ }^{\mathrm{b}}$ and \\ Cristín Ryan MPharm PhD MRPharmS ${ }^{\mathrm{c}}$
}

a PhD Candidate, School of Pharmacy, Queen's University Belfast, Belfast, UK b Professor of Primary Care Pharmacy, Queen's University Belfast, Belfast, UK c Lecturer in Clinical Pharmacy, Queen's University Belfast, Belfast, UK

\begin{abstract}
Rationale, aims and objectives: Intermediate care (IC) describes a range of services targeted at older people, aimed at preventing unnecessary hospitalisation, promoting faster recovery and maximising independence. The introduction of IC has created a new interface between primary and secondary care. Older people are known to be at an increased risk of medication-related problems when transferring between healthcare settings and pharmacists are often not included as part of IC multidisciplinary teams. This study aimed to explore community pharmacists' (CPs) awareness of IC services and to investigate their views of and attitudes towards the medicines management aspects of such services, including the transfer of medication information.

Method: Semi-structured interviews were conducted, recorded, transcribed verbatim and analysed using a constant comparative approach with CPs practising in the vicinity of IC facilities in Northern Ireland, UK.

Results: Interviews were conducted with 16 CPs. Three themes were identified and named 'left out of the loop', 'chasing things up' and 'closing the loop'. CPs felt that they were often 'left out of the loop' with regards to both their involvement with local IC services and communication across the healthcare interfaces. As a result, CPs resorted to 'chasing things up' as they had to proactively try to obtain information relating to patients' medications. CPs viewed themselves as ideally placed to facilitate medicines management across the healthcare interfaces (i.e., 'closing the loop'), but several barriers to potential services were identified.

Conclusion: CPs have limited involvement with IC services. There is a need for improvement of effective communication of patients' medication information between secondary care, IC and community pharmacy. Increasing CP involvement may contribute to improving continuity of care across such healthcare interfaces, thereby increasing the person-centeredness of service provision.
\end{abstract}

\section{Keywords}

Community pharmacists, continuity of care, information transfer, intermediate care, medicines management, older people, person-centered healthcare, pharmacy, qualitative study

\section{Correspondence address}

Ms. Anna N. Millar, School of Pharmacy, Queen’s University Belfast, 97 Lisburn Road, Belfast, BT9 7BL, UK.

E-mail: anna.millar@qub.ac.uk

Accepted for publication: 6 July 2015

\section{Introduction}

Intermediate care (IC) is a care setting that has evolved over a number of years in response to the ageing population, the increasing pressure faced by acute healthcare services and the resulting need for alternatives to hospital-based care. Although the term 'IC' originated in the United Kingdom (UK), other countries have adopted similar strategies; several equivalent healthcare models with similar objectives to IC services are used globally and are denoted by a variety of terminologies including 'subacute care', 'post-acute care' and 'transition care' [1]. IC has been broadly defined in the $\mathrm{UK}$ as ' $a$ range of integrated services to prevent unnecessary hospital admission, promote faster recovery from illness, support timely discharge and maximise independent living' [2]. Alongside the rest of the UK, Northern Ireland (NI) now has numerous services that fall under the umbrella term of IC; such services are provided in a range of settings including, but not limited to, nursing and residential care homes and have the potential to increase the personcenteredness of care provision.

The introduction of IC as a healthcare setting between primary and secondary care has created an additional interface across which medicines have to be managed. Receiving care in numerous settings is a recognised risk factor for discontinuity of care in relation to medicines 
management [3]. An integral component of IC is the involvement of a multidisciplinary team to meet patients' care needs [2]. Additionally, cross-professional working has been credited with improving the continuity of patient care at the point of healthcare interface transitions [4]. Pharmacists have a defined role in medicines management (ensuring the safe and effective use of medicines by patients) within both primary and secondary care settings, yet their role in relation to the IC setting remains unclear.

Previous work [5] has suggested that communication is minimal or non-existent between IC facilities and Community Pharmacists (CPs) on discharge. The resulting discontinuity of care will have implications for the CPs who care for such patients on their return to the community. The aim of this study was therefore to explore CPs' awareness of IC services provided in their local area and to investigate CPs' views of and attitudes towards the medicines management aspects of such services including the transfer of medication information between healthcare settings.

\section{Methods}

This study adopted a qualitative approach, employing semi-structured interviews. The focus of the interview schedule was informed by the results of the previous work [5] which highlighted issues surrounding CPs' involvement in IC, the supply of medicines to IC via community pharmacies and the transfer of information to patients' CPs when admitted to IC and also at discharge. The content of the interview schedule is detailed in Box 1. Pilot interviews were conducted (with experienced researchers and practising pharmacists) to test the interview schedule for clarity and it was refined accordingly.

\section{Box 1 Semi-structured interview schedule content}

- $\quad$ CPs' level of awareness and involvement with IC services $>$ Awareness of the role of IC within the wider health service context.

Involvement with services provided to IC patients by CPs.

$>$ Views on integration with local IC services

- CPs' views on the transfer of medication information when patients transfer between healthcare settings.

> Provision of discharge information from hospital/IC facilities.

$>$ Satisfaction with the communication of such information

Initially, CPs were identified and purposively sampled through the network of connections established prior to the present study, whereby IC facility managers aided in identifying community pharmacies. In conjunction, a participant recruitment strategy of 'snowball' sampling was employed [6]. This approach facilitated the identification of participants who may not have been identified using only the purposive sampling approach [7]. Data analysis was conducted in parallel to data collection. Recruitment ceased at the point when data saturation was deemed to have occurred, noted by the absence of new emergent themes.

Data were transcribed verbatim, all identifiers were removed and codes assigned to participants. Data were imported to NVivo ${ }^{\circledR}$ to facilitate analysis using a constant comparative approach, involving the simultaneous coding and analysis of the data in order to develop and refine themes and explore their relationships to one another [8]. Emergent themes were then coded alongside supporting verbatim quotes. The initial analysis was completed by one researcher, with a random sample of transcripts subjected to the same analysis by a second researcher. Consensus on the emergent themes was reached by discussion among all 3 researchers. Ethical approval was granted for the study by the School of Pharmacy Ethics Committee, Queen's University Belfast, UK.

\section{Results}

Interviews were conducted with $16 \mathrm{CPs}$, of whom 10 (62.5\%) were female. Eleven (68.8\%) were from independent pharmacies and 5 (31.3\%) were employed within community pharmacy chains. The mean number of years qualified was 14 years (range 2-35 years). Table 1 details the demographic information pertaining to all participants.

\section{Left out of the loop}

Most CPs demonstrated some understanding of the IC concept, though few were able to name IC facilities in their local area or distinguish between IC facilities and other non-IC facilities, such as traditional nursing homes. Furthermore, it was evident that the terminology used to describe such services lacked clarity amongst CPs, as many incorrectly referred to these services as 'respite':

“... [Intermediate care] seems like a newer term, maybe just a replacement term for respite. Respite is a bit scary a term... it sounds a bit terminal, respite, whereas intermediate means it is short term and you are going to come out.” (CP9)

"I have never heard [intermediate care facility] referred to as anything apart from respite.” (CP8)

The CPs who were aware of both the IC concept and specific local IC facilities attributed this to their direct involvement with the facility(s) throughout the course of their practice: 
Table 1 Demographic data of community pharmacists recruited to the study

\begin{tabular}{lllll}
\hline $\begin{array}{l}\text { Pharmacist } \\
\text { Code }\end{array}$ & $\begin{array}{l}\text { Gender } \\
\text { (Male/Female) }\end{array}$ & Years qualified & Position & $\begin{array}{l}\text { Type of pharmacy (Multiple } \\
\text { or Independent) }\end{array}$ \\
\hline 1 & Male & 3 & Pharmacy Manager & Multiple \\
\hline 2 & Female & 2 & Pharmacist & Independent \\
\hline 3 & Female & 7 & Pharmacist & Independent \\
\hline 4 & Female & 10 & Pharmacy Manager & Multiple \\
\hline 5 & Female & 7 & Pharmacy Manager & Independent \\
\hline 6 & Male & 23 & Pharmacy Owner & Independent \\
\hline 7 & Female & 7 & Pharmacist & Independent \\
\hline 8 & Male & 35 & Pharmacy Owner & Independent \\
\hline 9 & Female & 9 & Pharmacy Manager & Multiple \\
\hline 10 & Female & 21 & Pharmacy Manager & Independent \\
\hline 11 & Male & 18 & Pharmacy Manager & Independent \\
\hline 12 & Male & 5 & Pharmacy Manager & Independent \\
\hline 13 & Female & 18 & Relief Pharmacist & Multiple \\
\hline 14 & Female & 11 & Pharmacy Manager & Multiple \\
\hline 15 & Female & 17 & Pharmacy Manager & Independent \\
\hline 16 & Male & 30 & Pharmacy Owner & Independent \\
\hline
\end{tabular}

“... [CPs] probably just assume [IC facilities] are just nursing homes so they don't realise that there's actually a difference. I didn't really know about them until I worked in that specific pharmacy.” (CP4)

When asked to describe the extent of their involvement with IC facilities, all the CPs reported having minimal, if any, contact or input with local IC services:

"We don't deliver and we don't interact and I don't know any contacts in either [local IC facility].” (CP3)

“...the meds [sic] are normally organised between the hospital and wherever it is they're going usually, we are not really involved.” (CP9)

With regard to the transfer of patients' medication information across the healthcare interfaces, despite articulating predominantly negative views, the majority of CPs described a general trend of improvement:

"I have seen an improvement in recent years, [communication] used to be really awful.” (CP1)

Despite the overall positive trend, CPs also noted that they regularly encountered instances where there was inadequate or non-existent communication across the healthcare interface. The information contained within discharge letters was deemed to be sufficiently detailed when received. However, CPs reported that with many patients they are 'left out of the loop' with regards to the communication of medication information:

"It's variable; sometimes you will get a phone call, sometimes you will not, most times you will not. Sometimes you will get a letter, sometimes you do not and most times you do not." (CP16)
In addition to gaps in the provision of communication relating to patients' medications, several CPs also commented that they were 'left out of the loop' when their patients were admitted to hospital or IC. It would appear that it was often the case that CPs were only made aware of a patient's admission or discharge after the transition had already occurred and frequently as a result of chance contact with the patient's family, instead of via communication channels with fellow healthcare professionals:

"[Communication] varies from patient to patient, sometimes it all works seamlessly and you'd be kept in the loop...other times, the patient's representative comes in, that's the first you know of it and they're coming in looking medication and you've no record of any medication changes or discharge letter.” (CP11)

The unpredictable nature of communication, specifically between CPs and other healthcare professionals, had resulted in CPs feeling that they were systematically 'left out of the loop' with regard to information that they would consider pertinent.

\section{Chasing things up}

As a direct consequence of being 'left out the loop', CPs described how they assumed responsibility for remedying the absence of communication through a set of actions referred to as 'chasing things up'. In the absence of it being provided to them, CPs had to access such information via other healthcare professionals as currently they do not have access to patients' medical records. Consequently, several CPs called for increased autonomy for CPs' access to information: 
“...in a lot of cases [communication] is non-existent and it takes us an awful lot of time chasing up records that we should have instant access to, ideally we should be linked in with the ECR [electronic care record].” (CP6)

CPs were found to be generally unsatisfied with the current modes of communication used by secondary care and IC services to facilitate the transfer of information across the healthcare interface. At discharge, a longstanding approach adopted by secondary care services involved entrusting the patient (or their representative) to deliver a discharge summary to the patient's GP and, occasionally, the patient's CP. Such an approach was viewed to be an unreliable method of communication by CPs and often patients would confuse such documents as prescriptions:

\begin{abstract}
"It's a paper-based transfer in a day of IT (information technology) and in some cases it will go through a family member, who is unaware of the requirements...so a pharmacist faced on a Saturday morning for example, with a hospital discharge letter is left in a very difficult position because... the legal issue is quite simple, there's no prescription, there's no medicine.” (CP16)
\end{abstract}

It was also observed that the lack of communication was not limited to secondary care and IC, but also extended to other healthcare settings, including primary care. Again, the lack of consistent and timely communication engendered feelings of frustration as CPs concluded they were often the 'forgotten' healthcare profession. The communication between CPs and their GP counterparts was seen as being particularly challenging:

"The [discharge] letter gets sent to the GP, put on their
records, but we don't find out until we go to request a
new prescription.... a month later...they never think to
inform the community pharmacist of changes.” (CP7)

"You just presume that...there would be some sort of interaction...the GPs should be telling you that 'this patient's taken unwell' or even 'that patient's died', and you are sitting ordering away scripts and nobody tells you, yeh I think that's a bit strange.” (CP3)

As previously alluded to, CPs described how they were often left to make challenging ethical decisions as a result of demands from other healthcare professionals and patients alike. Several CPs described occasions involving both hospitals and IC facilities, where they were requested to dispense medications for patients following discharge without the prior authority of a prescription. The resultant pressure to obtain a prescription from the GP at short notice often fell to the $\mathrm{CP}$, causing feelings of resentment and frustration:

"We were phoned there recently from [IC facility] to say 'we are letting the patient out without anything' and you're like 'What? Wise up!' And they're like 'no they're not getting anything, can you deal with it?' [Sighs] We have to phone the doctors, it's daft. We have to make sure they've got the email from the hospital to get the prescription, set up the new tablets, get the new tablets sent up to the [IC facility].” (CP10)

"Legally we are supposed to have a doctor's prescription and like [the hospital staff] are basically trying to force it on you, which I don't think is right.” (CP11)

CPs portrayed themselves as intermediaries between hospitals, IC facilities and GP surgeries as responsibility for 'chasing things up' fell to them. Although they deemed it unavoidable, CPs viewed time spent engaging in such activities as a misuse of their time:

"We can chase [GPs] up and run after them, but it shouldn't really be our responsibility and it does increase our workload.” (CP5)

\begin{abstract}
"It will always come down to us chasing because we are the ones on the cold front, we are the ones that are providing the medication so we have to have the prescription to do it, so if we don't chase it and there's no prescription then there's no medication for the person.” (CP9)
\end{abstract}

Another example of a challenge encountered by CPs related to situations where GPs failed to update patient records to reflect changes to patients' medications regimens made in secondary care or IC. Subsequently, CPs were confronted with prescriptions, issued by GPs, which were not consistent with the patient's discharge letter, again giving rise to CPs resolving to 'chase' the GPs in order to reconcile the two:

"Sometimes there are discrepancies there definitely and I find that quite a lot between what the GP has written and what we have been told and seen from the hospital letter. Therefore we are the ones that need to intervene... we kind of seem to be in the middle of that”. (CP15)

In general, CPs did not attribute blame to individual GPs, but rather to the way in which discharge letters were handled within the GP's surgery. Several CPs voiced concerns that the responsibility for updating patients' medication records lay with reception staff in GP surgeries:

"[Discharge letters] are handled by a receptionist ...they're not well enough trained and not well enough qualified to pick all those things up, where they see a drug name and just click the button, repeat that medication, the dose and timing of it may have changed.” (CP6)

\section{Closing the loop}

The majority of CPs responded positively to the suggestion of increased involvement with IC services. Participants viewed CPs as being ideally placed to facilitate patients' continuity of care during a stay in IC and ensuring medicines were managed safely whenever a patient was transferred between healthcare settings. Several CPs viewed such involvement as an opportunity that would 
facilitate the profession's development towards roles utilising pharmacists' unique skills:

\begin{abstract}
"I would like to be involved because it would give you an opportunity to get out of what can become kind of laborious, just checking prescriptions and it would give you a feeling of being part of a team, being in that group of professionals that would be looking after [patients]." (CP1)

“...that's what to me community pharmacy is all about...speaking to the patients and trying to help them and definitely if there was a scheme like that it would be really interesting and you are putting your clinical skills and your clinical knowledge to use rather than checking boxes in the dispensary." (CP7)
\end{abstract}

Aside from such services typically offered to nursing homes by CPs, such as the supply of medications, the majority of respondents then went on to identify new roles, specific to the IC setting that could be developed. Several CPs commented that a patient's stay in IC represented an ideal opportunity for CPs to provide medication counselling:

"[Pharmacists] could offer advice to the patients...a lot of those patients don't even know what meds [sic] they are on, so if they are coming out of hospital on new meds although they agree with the doctor and consultant in the hospital at the time, they mightn't know what the meds are for..." (CP12)

CPs also described their views on providing a service for IC patients which would include a pharmacistconducted review of the appropriateness of patients' medications:

"You'd be...looking at the appropriateness of the [medications] they've brought in and ensuring they get the right stuff." (CP3)

When considering potential roles for CPs within the IC setting, many referred to the concept of having a single point of contact who advocated for the patient when they were transitioning between healthcare settings. This person would have responsibility for ensuring the continuity of care in relation to medicines, specifically with regard to communication with relevant stakeholders on both sides of the interface. CPs saw this approach to service provision as more effective use of a pharmacist, rather than having multiple pharmacists visiting facilities for their respective patients.

"It would just be great to get a system in place... somebody assigned to making sure the discharge information is passed right down the chain and we are not forgotten about.” (CP7)

When asked what barriers existed to the development of any community pharmacy service being introduced in an IC setting, remuneration was at the forefront of all the interviewees' responses. Additionally, some CPs stated that they were already involved with numerous services and therefore would not have the time to facilitate further services:

"With all the new services...I think we are taking on too much...but at the same time...we also need to be pushing to get as much as we can because clearly the dispensing fee is going down.” (CP15)

"If there were payment in place for those things, I think most pharmacists would be happy to do it.” (CP14)

The majority of CPs also commented that a transformation of the existing community pharmacy remuneration structure in NI would need to occur in order for such services to realise their full potential:

“...if pharmacists are continually...paid to dispense, then you are going to get a dispensing service and that's what the health service has got, so there is no bigger barrier... I don't think there's any lack of ambition, I think the issue is purely a contractual issue in terms of what is paid for." (CP16)

To a lesser extent, issues surrounding inter- and intraprofessional rivalry were seen by some as potential barriers to integration with IC services:

"There's a certain amount of interprofessional rivalry, there's GPs who wouldn't necessarily want us to get involved.” (CP6)

"...there is a good relationship [with GPs] and you wouldn't want to put anybody's nose out of joint.” (CP2)

Despite these highlighted barriers, the majority of CPs agreed that any service targeted at improving continuity of care would be beneficial not only for their profession as a whole, but ultimately for their individual patients who were increasingly receiving care across numerous healthcare settings.

\section{Disc ussion}

This study is, to the best of the authors' knowledge, the first of its kind to describe CPs' awareness of and involvement with IC services. CPs were found to have a limited understanding of the concept of the IC model and many did not differentiate between IC and other similar, yet conceptually different services, such as residential care services and respite care. It could be hypothesised that this lack of awareness is, in part, the result of a lack of communication to CPs about IC, as services have evolved. Whilst still considered a relatively new concept, there were, at the time of the research, numerous established IC services in NI. Despite this, confusion as to what does and what does not constitute IC still remained. The debate surrounding the terminology used has been previously described by the authors [5]. Perhaps unsurprisingly, CPs who worked in a pharmacy that regularly supplied 
medicines to patients in IC facilities were more familiar with the concept of IC compared with those who did not.

Another aim of this study was to explore CPs' views on the communication of information relating to patients' medications across the various healthcare interfaces. Similarly in this context, pharmacists felt 'left out of the loop' with regards to communication between healthcare settings. During an episode of care in hospital, patients' medication regimens are often the subject of change as existing medications may be stopped, doses may be altered, or new medications may be added [9]. Despite noting a tangible improvement in communication, CPs recognised that there were still significant gaps in communication between secondary care, IC and primary care. Effective communication is regarded as a fundamental component of successful transitional care, a term used to describe the processes which achieve continuity of care as patients transfer between different healthcare settings [3]. CPs, although satisfied with the level of detail contained in discharge letters, found the inconsistent nature with which such communications were received to be the limiting factor affecting patients' transitional care.

A questionnaire study by Munday et al. [10] found that the majority (94\%) of CPs were in favour of being informed of changes made to patients' medication regimens upon discharge from secondary care. Therefore, CPs may feel as though they have been 'left out of the loop' when such information is not communicated to them. Notwithstanding the resultant inconvenience to CPs, the importance of receiving such communication cannot be discounted from a patient safety perspective. The increased risk of medication discrepancies occurring at healthcare interfaces is widely recognised. Forster et al. [11] reported that of 581 hospitalised patients, nearly one in 5 experienced an adverse event during their subsequent transition from the hospital to home. Of these, two-thirds were adverse drug events. It therefore follows that if CPs do not receive communication relating to patients' medication regimens, then the potential for such errors to reach the patient is increased. At present, CPs in NI cannot independently access patients' medical records or discharge letters; the latter may be provided to CPs at the discretion of the discharging hospital or IC facility. A review by Kripalani et al. [12] highlighted how traditional discharge summary communication methods (such as postal delivery or delivery via the patient) are suboptimal for communicating information in a timely and accurate manner to healthcare providers responsible for the continuing care of the patient following discharge.

As a direct consequence of insufficient communication, it would appear that the act of 'chasing things up' has become the standard practice of CPs. This theme described the routine adopted by CPs of pursuing other healthcare professionals (primarily GPs) in order to obtain either discharge summaries and/or prescriptions. It was clear that CPs felt compelled to participate in this perpetual cycle in order to fulfil what they viewed as a professional obligation: the reconciliation of patients' medications. As a result, CPs have assumed the role of intermediaries at the interfaces of care. While perhaps traditionally seen as the GP's role, it is evident that CPs are increasingly taking a proactive stance to ensure continuity of care for their patients, a key element of person-centered healthcare.

CPs, in general, considered 'chasing things up' as a time-consuming and unappreciated task. Communication between community pharmacies and other healthcare settings appears to be largely dependent upon outdated methods such as handwritten letters, delivered by patients or their representatives. Modernisation of the methods of communication to reflect current available technologies such as the electronic care record need to be extended to community pharmacy settings in order to help bridge the gap across the healthcare interfaces.

The issue of patients misinterpreting discharge letters for prescriptions has been highlighted as a cause for concern. It is important that patients are encouraged to take an active role in the management of their medicines, again a key element of person-centered healthcare, hence many hospitals in NI now provide patients with their own medication record summary on discharge. However, it would appear that continued effort is required to ensure patients are educated in the purpose of such information.

Importantly, many CPs had strong views on communication, or lack thereof, from GPs. Indeed, by characterising the pharmacist-GP interaction as 'chasing', it would imply that CPs believe GPs are not voluntarily communicating information. Whilst this may be true in some cases, it is also possible that the GPs themselves are not receiving effective communication in a timely manner from their hospital or IC counterparts [13,14]. A survey, conducted in 2000, found that two-thirds of GPs have experienced delays in receiving discharge information and 93\% of CPs had encountered instances where patients presented to the pharmacy requesting medicines following their discharge without having obtained a prescription from their GP [15].

Many of the CPs interviewed attributed the examples of communication failures emanating from GP practices to receptionists working within those practices. Crowe et al. [13] have also described similar findings where administrative staff's inability to interpret and therefore reluctance to act upon, information from discharge summaries, may lead to errors in patients' medication records. The same study, which looked at how information is processed within GP practices, described how GPs may not always even be aware when one of their patients is admitted to hospital, suggesting that it is therefore not only CPs who may be 'left out of the loop' in regards to communication across the healthcare interfaces.

The importance of ensuring continuity of care across healthcare interfaces cannot be overstated. More than half of all medication errors occur during transitions of care, when responsibility is passed between healthcare professionals [16]. It therefore follows that the more interfaces a patient transitions through in one episode of care, the more likely it is that they will experience a medication error as a result. Furthermore, efforts to optimise patients' medication regimens are made redundant if such changes are 'lost in transition'. To help avoid such situations, it is important that discharge 
summaries and/or letters contain sufficient detail on the reasons for changes to medications to reduce the likelihood of practitioners in primary care interpreting any change as accidental and therefore adding or omitting drugs or amending doses. Despite this, a large scale survey of hospital discharges reported that a minority of hospitals routinely communicate with CPs during the discharge process with contact being made with CPs, on average, for only $5.1 \%$ of patients [17]. If the desired standard of seamless care is to be realised in health services, it is imperative that, where necessary, CPs are consistently communicated with by hospitals and IC facilities.

The results of this study demonstrate how pharmacists view themselves as ideally placed to provide IC services to patients. The ambition continually to expand CPs' roles to beyond the dispensary is not a new one, as the profession is increasingly seeking new areas of practice into which it can integrate [18]. CPs' existing transferable skills could be used to enhance medicines management across the healthcare interfaces. For instance, a patient's stay in IC represents an ideal opportunity for pharmacists to provide medication counselling. Patients' medication regimens may have changed as a result of their earlier hospital admission and so time spent in an IC facility, under the supervision of healthcare workers, can be used to allow patients to adapt to a new medication regimen, thus optimising their ability to confidently remain as independent as possible with regard to the management of their own medicines [19].

A potential service that could be implemented by CPs to facilitate continuity of care at discharge is that of the liaison pharmacist role. A recent pilot study investigated the effect of instigating a hospital-based liaison pharmacist programme which was subsequently shown to improve the accuracy of drug histories, improve patients' knowledge about their medications and reduce the rate of discrepancies between hospital discharge letters and medicines subsequently dispensed in the community [20].

This study also highlighted that the main barriers identified by CPs that would hinder their involvement in any new service were the time required to deliver the service and the expected remuneration for providing the service. The majority of CPs were only interested in being involved in services that they viewed as being potentially profitable. Interprofessional rivalry was also identified as a minor barrier to the development of a new service. CPs were concerned that being more actively involved in healthcare settings beyond the community pharmacy may potentially jeopardise their existing professional relationships with GPs. Similar concerns have been described previously; qualitative work by Hughes and McCann [21] has revealed that interprofessional barriers between pharmacists and GPs stem from the perceived 'shopkeeper image' which may be seen as being a conflict of interest in a healthcare setting. As the face of the pharmacy profession changes, it is to be expected that there will be a blurring of the boundaries between healthcare professionals and their traditional roles. Multidisciplinary training at both undergraduate and postgraduate level has been suggested as a potential solution to help overcome these interprofessional barriers [22].

While we found that CPs were generally positive towards using their expertise in more ways and moving away from the traditional roles associated with community pharmacy practice, it was also evident that the undertaking of such roles is primarily motivated from a 'business' point of view. Perhaps the community pharmacy model must change to one that rewards CPs' abilities to improve patients' health outcomes before the integration of community pharmacy within IC services and at the healthcare interface can be realised.

This was the first study of its kind to report on CPs and the IC setting. As it was qualitative in nature, the results are not intended to be generalizable to all contexts. However, data saturation was achieved with our sample size. Finally, validation checks were conducted using a second researcher and consensus within the team on the final themes.

\section{Conclusion}

CPs in NI have limited awareness of and involvement with local IC services and are often left out of the communication loop when their patients move between care settings. Despite this, CPs are ideally placed as healthcare professionals to provide a range of services which could potentially improve patient-related outcomes by facilitating seamless care when patients are transitioning through the healthcare interfaces. Further work is required to explore the themes this study has raised and to evaluate how these affect the wider community pharmacy service through their potential to increase the person-centeredness of care.

\section{Acknowledgements and Conflicts of Interest}

This work was supported by the Department for Employment and Learning (DEL) Northern Ireland. The authors wish to thank all of those who gave their time to participate in this study. All authors disclose no conflicts of interest.

\section{References}

[1] Millar, A.N., Hughes, C.M., Passmore, A.P. \& Ryan, C. (2014). Intermediate Care: The Role of Medicines Management. Drugs and Aging 31 (1) 21-31.

[2] Department of Health. (2009). Intermediate CareHalfway Home- Updated Guidance for the NHS and Local Authorities. Leeds: Department of Health.

[3] Coleman, E. (2003). Falling through the cracks: Challenges and opportunities for improving transitional care for persons with continuous complex care needs. Journal of the American Geriatrics Society 51 (4) 549-555. 
[4] Vaughan, B. \& Lathlean, J. (1999). Intermediate care: models in practice. London: King's Fund.

[5] Millar, A.N., Hughes, C.M. \& Ryan, C. (2015). "It's very complicated": A qualitative study of medicines management in intermediate care facilities in Northern Ireland. BMC Health Services Research In press.

[6] Coyne, I. (1997). Sampling in qualitative research. Purposeful and theoretical sampling; merging or clear boundaries? Journal of Advanced Nursing 26 (3) 623-630. [7] Marshall, M. (1996). Sampling for qualitative research. Family Practice 13 (6) 522-525.

[8] Bradley, E.H., Curry, L.A. \& Devers, K.J. (2007). Qualitative data analysis for health services research: Developing taxonomy, themes, and theory. Health Services Research 42 (4) 1758-1772.

[9] Cochrane, R., Mandal, A., Ledger-Scott, M. \& Walker, R. (1992). Changes in Drug-Treatment After Discharge from Hospital in Geriatric-Patients. British Medical Journal 305 (6855) 694-696.

[10] Munday, A., Kelly, B., Forrester, J.W.E., Timoney, A. \& McGovern, E. (1997). Do general practitioners and community pharmacists want information on the reasons for drug therapy changes implemented by secondary care? British Journal of General Practice 47 (422) 563-566.

[11] Forster, A., Murff, H., Peterson, J., Gandhi, T.K. \& Bates, D.W. (2003). The incidence and severity of adverse events affecting patients after discharge from the hospital. Annals of Internal Medicine 138 (3) 161-167.

[12] Kripalani, S., LeFevre, F., Phillips, C.O., Williams, M.V., Basaviah, P. \& Baker, D.W. (2007). Deficits in communication and information transfer between hospitalbased and primary care physicians - Implications for patient safety and continuity of care. Journal of the American Medical Association 297 (8) 831-841.

[13] Crowe, S., Tully, M.P. \& Cantrill, J.A. (2010). Information in general medical practices: the information processing model. Family Practice 27 (2) 230-236.

[14] Penney, T. (1988). Delayed communication between hospitals and general practice: Where does the problem lie? British Medical Journal 297 (6640) 77-82.

[15] Brookes, K., Scott, M. \& McConnell, B. (2000). The benefits of a hospital based community services liaison pharmacist. Pharmacy World \& Science 22 (2) 33-38.

[16] Rozich, J. \& Resar, R. (2001). Medication safety: one organization's approach to the challenge. Quality Management in Health Care 8, 27-34.

[17] Sexton, J., Ho, Y., Green, C. \& Caldwell, N.A. (2000). Ensuring seamless care at hospital discharge: a national survey. Journal of Clinical Pharmacy and Therapeutics 25 (5) 385-393.

[18] Edmunds, J. \& Calnan, M.W. (2001), The reprofessionalisation of community pharmacy? An exploration of attitudes to extended roles for community pharmacists amongst pharmacists and General Practioners in the United Kingdom. Social Science \& Medicine 53 (7) 943-955.

[19] Al-Rashed, S., Wright, D., Roebuck, N., Sunter, W. \& Chrystyn, H. (2002) The value of inpatient pharmaceutical counselling to elderly patients prior to discharge. British Journal of Clinical Pharmacology 54 (6) 657-664.
[20] Bolas, H., Brookes, K., Scott, M. \& McElnay, J. (2004). Evaluation of a hospital-based community liaison pharmacy service in Northern Ireland. Pharmacy World \& Science 26 (2) 114-120.

[21] Hughes, C. \& McCann, S. (2003). Perceived interprofessional barriers between community pharmacists and general practitioners: a qualitative assessment. British Journal of General Practice 53 (493) 600-606.

[22] Carlisle, C., Cooper, H. \&Watkins, C. (2004). "Do none of you talk to each other": the challenges facing the implementation of interprofessional education. Medical Teacher 26 (6) 545-552. 